\title{
Postcoital bleeding in a case of recto-vaginal endometriosis.
}

\author{
Haitham A Torky*, Ashraf Abo-Louz \\ Department of Obstetrics and Gynecology, October 6th University, Giza Governorate, Egypt
}

\begin{abstract}
Introduction: Rectovaginal Endometriosis (RVE) is a severe form of endometriosis, less frequent than peritoneal or ovarian endometriosis. Recto-vaginal endometriosis has multiple diagnostic and management options with long-term outcomes varying according to the management strategy used. Case: A 40 years old woman complained of postcoital bleeding. Detailed history revealed that she suffered from persistent dysmenorrhea, dyschasia and constipation. Vaginal speculum examination revealed a large fleshy polypoidal mass in the posterior vaginal fornix, which was excised and biopsied. Histo-pathological examination revealed vaginal endometriosis. Four months later the mass recurred, in spite of, receiving hormonal treatment. Ultrasound and MRI revealed multiple uterine fibroids, with heterogenous lesion located in the rectovaginal area, and CA-125 was $80 \mathrm{u} / \mathrm{ml}$. exploratory laparotomy was done after proper bowel preparation. Total abdominal hysterectomy with excision of the upper vaginal portion and a part of the anterior aspect of the recto-sigmoid was resected due to the invasion of its musculosa, short of the mucosa, with primary repair. Histo-pathological examination showed advanced endometriosis including the vaginal fornix and the rectal wall. The patient received two doses of Leuprolide Acetate $\mathbf{1 1 . 2 5}$ mg as an adjuvant treatment. Conclusion: All women with postcoital bleeding or pelvic pain should have a detailed history taken from them and thorough examination including speculum examination and visualization of posterior vaginal fornix to detect vaginal endometriosis. The primary line of management for recto-vaginal endometriosis is surgery, as hormonal therapy is less effective in such cases, which might be explained by difference in origin from the peritoneal disease.
\end{abstract}

Keywords: Postcoital bleeding, Recto-vaginal endometriosis, Surgical treatment.

Accepted on August 21, 2017

\section{Introduction}

Rectovaginal Endometriosis (RVE) is a severe form of endometriosis classified by Kirtner as stage 4 [1,2]. It is less frequent than peritoneal or ovarian endometriosis affecting $3.8 \%$ to $37 \%$ of patients with endometriosis $[3,4]$. RVE infiltrates the rectum, vagina, and rectovaginal septum, up to obliteration of the pouch of Douglas [4]. Endometriotic nodules exceeding 30 $\mathrm{mm}$ in diameter have $17.9 \%$ risk of ureteral involvement [5], while $5.3 \%$ to $12 \%$ of patients have bowel endometriosis, most commonly found in the recto-sigmoid involving $74 \%$ of those patients $[3,4]$.

Preoperative diagnosis can is challenging as there is absence of agreed upon disease-specific laparoscopic and radiological features, in spite of, studying several diagnostic tools as combined rectovaginal examination, transvaginal/transrectal ultrasounds, Computed Tomography (CT), Magnetic Resonance Imaging (MRI), colonography, colonoscopy, and, finally, laparoscopic removal with histological confirmation [6-8].

In RVE, medical treatments are often ineffective and have temporary effects in controlling symptoms. Complete surgical removal provides long-term pain relief and improve the quality of life, therefore making it the treatment of choice for most women $[1,4]$. Surgery consists of either excision or ablation through transvaginal, laparotomy, laparoscopy or combined approaches [4]. Commonly used techniques include shaving of rectal lesions, low anterior bowel resection and re-anastomosis, supericial laparoscopic Anterior Discoid Resection(ADR) [1].

Surgical treatment of bowel endometriosis is usually lengthy and technically challenging. Associated bowel surgery increases the risk of treatment complications up to 53\% [4]. Bowel resection is usually needed in $1.7 \%$ to $28.6 \%$ of cases [4].

Recto-vaginal endometriosis has multiple diagnostic and management options with long-term outcomes varying according to the management strategy used.

\section{Case}

40 years old para 2 (previous 2 C.S.) complained of post coital bleeding. Pap smear was done which revealed non-specific inflammation based upon the result she received several courses of antibiotics but didn't improve. Two months later she was referred to our clinic for consultation. Detailed history revealed that she suffered from persistent dysmenorrhea, dyschasia\& constipation. Vaginal speculum examination revealed a large fleshy polypoidal mass in the posterior vaginal fornix (Figure 1). Combined vaginal and rectal examination revealed a very vascular \& friable fleshy lesion, along with Left parametrial induration, and the mass was felt very close to the rectum with intact rectal mucosa. Based upon the above findings she was scheduled for removal of the polypoidal mass, in addition to, Dilatation and Curettage. Histo-pathological examination 


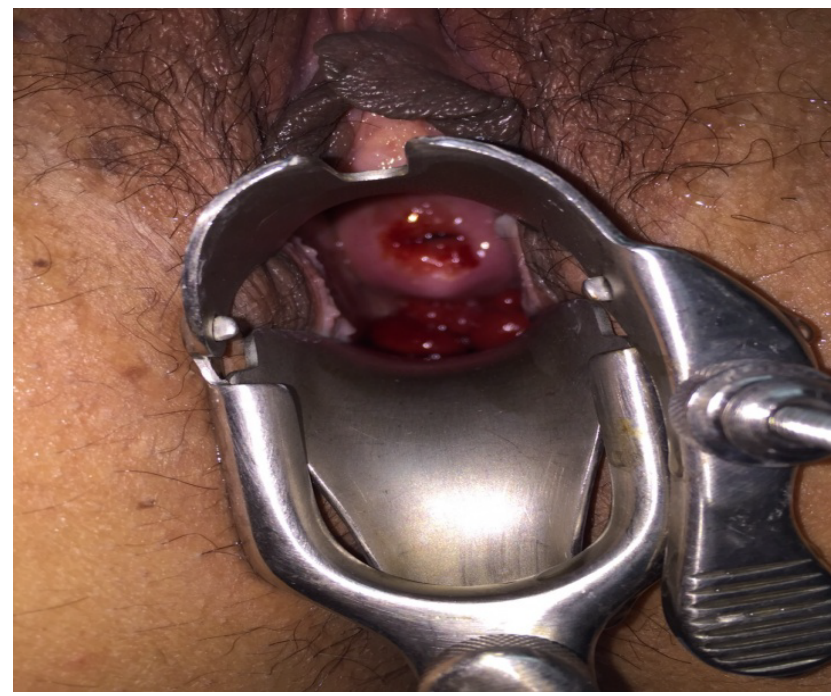

Figure 1. Showing endometriosis in the posterior vaginal fornix.

revealed vaginal endometriosis and endometrial hyperplasia, therefore, she was prescribed oral progesterone (Dienogest 2 $\mathrm{mg}$ ) continuous daily treatment; however, 4 months later during her treatment she developed dyspareunia, lower back and pelvic pain along with marked post coital bleeding. Combined vaginal and rectal examination showed a newly developed polyp in the posterior vaginal fornix, with left parametrial involvement, and a palpable mass in the rectovaginal area, with intact rectal mucosa, but inseparable from the posterior vaginal wall. Ultrasound and MRI revealed multiple uterine fibroids, with heterogenous lesion located in the rectovaginal area, and CA-125 was $80 \mathrm{u} / \mathrm{ml}$ so decision was taken for exploratory laparotomy after proper bowel preparation and the patient was consented for that. Total abdominal hysterectomy was done for multiple fibroids with preservation of both ovaries, in addition to, excision of the upper vaginal portion. A part of the anterior aspect of the recto-sigmoid was resected due to the invasion of its musculosa, short of the mucosa, with primary repair. The postoperative period went uneventful apart from a localized hematoma in the rectovaginal space which was managed conservative \& subsided spontaneously. Histopathological examination showed advanced endometriosis including the vaginal fornix and the rectal wall. The patient received two doses of Leuprolide Acetate $11.25 \mathrm{mg}$ as an adjuvant treatment.

\section{Discussion}

The current case is a case of extensive recto-vaginal endometriosis, which is believed to be different in both morphology and microscopy from peritoneal disease, and most probably arise from tracts of embryological remnants of Müllerian tissue inside the uterosacral ligaments, anterior bowel wall and rectovaginal septum [9-11]. There is variation in both estrogen and progesterone receptors when compared to eutopic endometrium, pointing out to differences in both regulatory mechanism and origin compared to peritoneal disease.

Symptoms include lower abdominal pain, severe backache, constipation and dyschezia [9], which were found in the current case. Speculum examination is useful in some cases and should focus on the posterior vaginal fornix, which showed a lesion in the current case. Vaginal endometriosis can present as disruption of the normal vaginal rugae, distortion, epithelial piling, small bluish cysts or a large reddish polypoid lesion as that found in the current case, therefore; excision biopsy was necessary in that lesion in order to confirm diagnosis. Combined rectal and vaginal palpation can confirm bowel involvement and in such cases bowel preparation should be done before surgery to allow for primary repair and avoid a possible colostomy, which was done before laparotomy in the current case.

Adamyan [12] classified recto-vaginal endometriosis into 4 stages which are: "Stage I: Endometriosis lesions are confined to the rectovaginal tissue in the area of the vaginal vault Stage II: Endometriosis tissue invades the cervix and penetrates the vaginal wall, causing fibrosis and small cyst formation. Stage III: Lesions spread into sacro-uterine ligaments and the rectal serosa. Stage IV: The rectal wall, recto-sigmoid zone and rectouterine peritoneum are completely involved, and the rectouterine pouch is totally obliterated".

Hormone manipulation is not effective in most cases, despite the presence of estrogen and progesterone receptors and symptoms usually recur after its stoppage [10,11]. Vercellini et al. [13] in their study concluded that medications achieved temporary quiescence of active lesions and can be used in selected cases, but surgery represented the definitive solution of such cases.

Surgical routes include traditional laparotomy, laparoscopy and recently, robotic surgery. Garry et al. [9] published the early analysis of the first 57 cases included in their study and concluded that surgery improves clinical symptoms and quality of life with acceptable operative morbidities. Chapron et al. [14] did a study to assess the efficacy of laparoscopic treatment of deep endometriosis involving 110 cases and concluded that in the hands of a skilled laparoscopic surgeon operative laparoscopy is efficient in treating pain related to deep endometriosis. Carvalho et al. [15] did a systemic review to evaluate the role of robotic surgery in the surgical treatment of deep endometriosis and concluded that this type of surgery is feasible without conversion; however, randomized controlled trials and studies assessing the long-term effects of robotic surgery are needed. 
Laparoscopic surgery performed by a skilled surgeon is considered the gold standard. The extent of resection is individualized. Most authors agree on superficial resection of the nodule or full disk resection of the rectal wall in case of a single nodule less than $3 \mathrm{~cm}$ in diameter, if bowel involvement is less than half its circumference. Bowel resection is indicated if it is not possible to resect the nodule or in cases with Adamyan stage IV, between 60 and $100 \%$ of such patients show symptom improvement [16]. Therefore; surgical en-bloc resection is the most efficient technique for treatment of obliteration of the cul-de-sac regardless of the route-as the technique used during laparotomy in the current case (Figures 2a and 2b) [17].

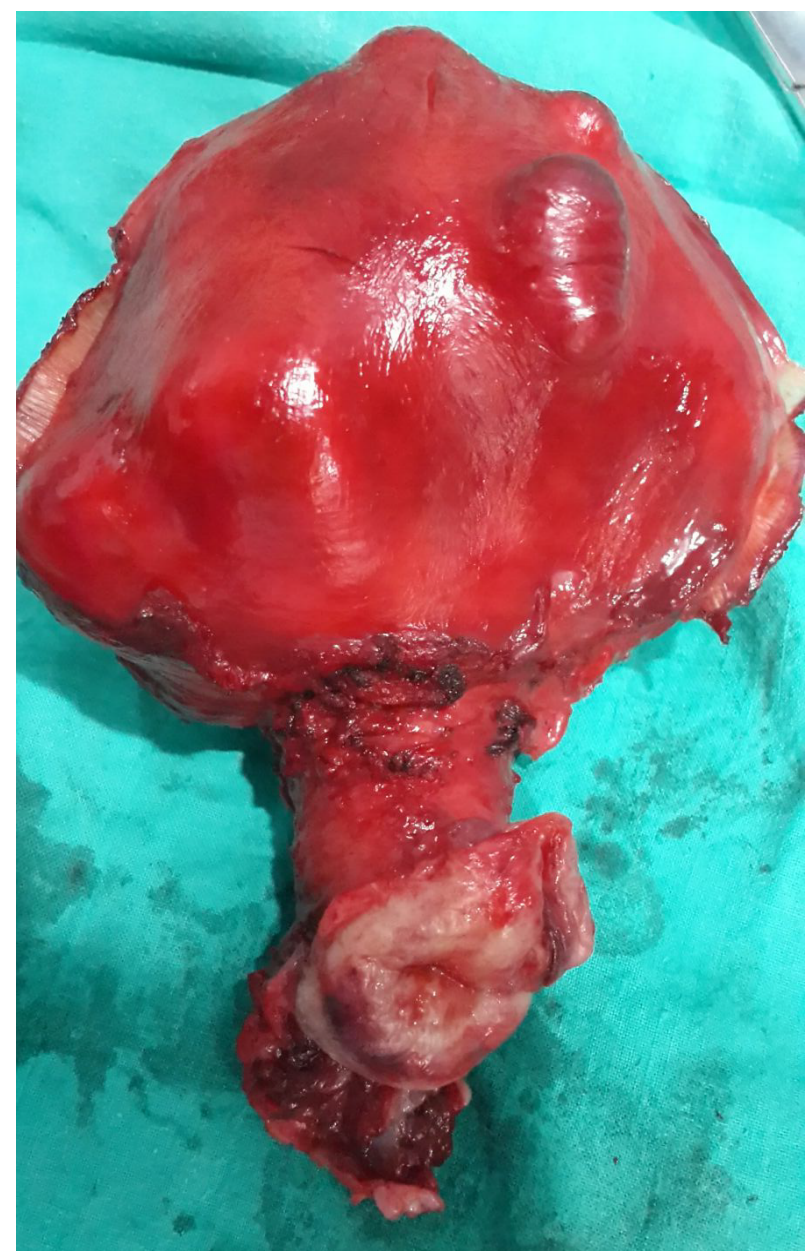

Figure 2a. Showing the hysterectomy specimen.

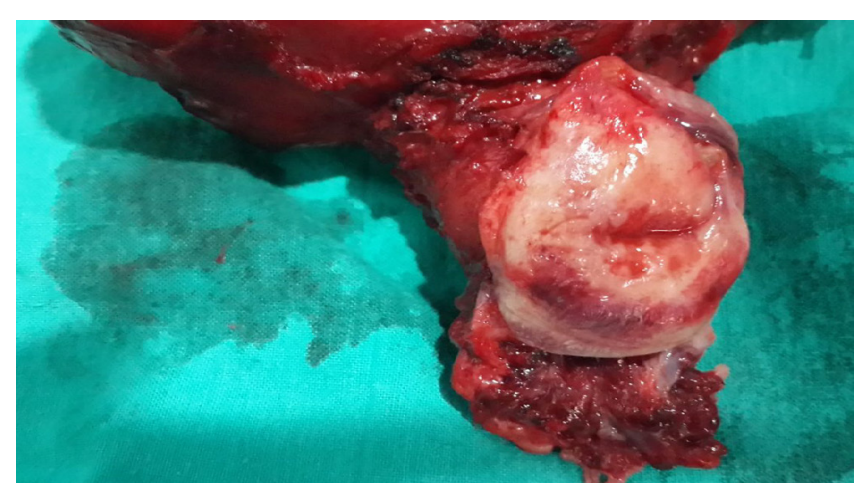

Figure 2b. Showing the cervix and the part of the removed part of the posterior vaginal wall containing endometriosis.

\section{Conclusion}

All women with postcoital bleeding or pelvic pain should have a detailed history taken from them and thorough examination including speculum examination and visualization of posterior vaginal fornix to detect vaginal endometriosis.

The primary line of management for recto-vaginal endometriosis is surgery, as hormonal therapy is less effective in such cases, which might be explained by difference in origin from the peritoneal disease.

\section{References}

1. Moawad NS, Guido R, Ramanathan R, et al. Comparison of laparoscopic anterior discoid resection and laparoscopic low anterior resection of deep infiltrating rectosigmoid endometriosis. JSLS. 2011;15(3):331-8.

2. Roberts CP, Rock JA. The current staging system for endometriosis: does it help? Obstet Gynecol Clin North Am. 2003;30(1):115-32.

3. Tarjanne S, Sjöberg J, Heikinheimo O. Rectovaginal endometriosis-characteristics of operative treatment and factors predicting bowel resection. J Minim Invasive Gynecol. 2009;16(3):302-6.

4. Zanetti-Dällenbach R, Bartley J, Müller C, et al. Combined vaginal-laparoscopic-abdominal approach for the surgical treatment of rectovaginal endometriosis with bowel resection: a comparison of this new technique with various established approaches by laparoscopy and laparotomy. Surg Endosc. 2008;22(4):995-1001.

5. Kondo W, Branco AW, Trippia $\mathrm{CH}$, et al. Retrocervical deep infiltrating endometriotic lesions larger than thirty millimeters are associated with an increased rate of ureteral involvement. J Minim Invasive Gynecol. 2013;20(1):100-3.

6. Abrao MS, Gonçalves MO, Dias JA, et al. Comparison between clinical examination, transvaginal sonography and magnetic resonance imaging for the diagnosis of deep endometriosis. Hum Reprod. 2007;22(12):3092-7.

7. Jelenc F, Ribič-Pucelj M, Juvan R, et al. Laparoscopic rectal resection of deep infiltrating endometriosis. J Laparoendosc Adv Surg Tech A. 2012;22(1):66-9.

8. Sassi S, Bouassida M, Touinsi H, et al. Exceptional cause of bowel obstruction: rectal endometriosis mimicking carcinoma of rectum-a case report. Pan Afr Med J. 2011;10:33.

9. Garry R, Clayton R, Hawe J. The effect of endometriosis and its radical laparoscopic excision on quality of life indicators. BJOG. 2000;107(1):44-54

10. Redwine DB. Mulleriosis: the single best fit model of origin of endometriosis. J Reprod Med 1988;33:915-20.

11. Halme J, Hammond MG, Hulka JF, et al. Retrograde menstruation in healthy women and in patients with endometriosis. Obstet Gynecol 1984;64(2):151-4.

12. Adamyan L. Additional international perspectives. In: 
Nichols DH (ed.) Gynecologic and Obstetric Surgery. St. Louis: Mosby Year Book. 1993;1167-82.

13. Vercellini P, Frontino G, Pietropaolo G, et al. Deep endometriosis: definition, pathogenesis, and clinical management. J Am Assoc Gynecol Laparosc 2004;11:153.

14. Chapron C, Dubuisson JB, Fritel X, et al. Operative management of deep endometriosis infiltrating the uterosacral ligaments. J Am Assoc Gynecol Laparosc. 1999;6:31-7.
15. Carvalho L, Abrão MS, Deshpande A, et al. Robotics as a new surgical minimally invasive approach to treatment of endometriosis: a systematic review. Int J Med Robot.

16. Foti N. Rectovaginal septum endometriosis. siccr-Italian society of colo-rectal surgery. 2012;33:270-4.

17. Redwine DB. Laparoscopic en bloc resection for treatment of the obliterated cul de sac in endometriosis. J Reprod Med 1992;37:695-8.

\section{*Correspondence to:}

Haitham A Torky

Department of Obstetrics and Gynecology October 6th University

Eygpt

E-mail: haithamtorky@yahoo.com 